\title{
A Design Method for Flexible Retaining Walls in Clay
}

\author{
C. Deng, S.K. Haigh, X. Ma, J. Xu
}

\begin{abstract}
$\underline{\text { ABSTRACT }}$
Design of retaining walls in clay is typically based on ultimate limit state calculations to prevent collapse with arbitrary factors of safety used to limit deformations. These factors of safety do not take into account the different rates of strength mobilisation in the wide variety of clays found worldwide. As there is substantial uncertainty in this approach, conventional design tends to lead to excessive conservatism with associated high cost. The novel analysis procedure based on the fraction of the strength of soil mobilised for a given wall displacement developed here allows rapid assessment of wall deformations and stresses via a simple two-parameter constitutive model which can be easily calibrated using conventional triaxial data. The model is validated based on field and model case histories with a variety of different clays and propping conditions and is shown to exhibit good performance in predicting the behaviour of published case histories based on soil parameters extracted from previously published soil test data. This novel analysis provides for the first time a route for practising engineers to carry out fast, efficient design at early stages of the design process by considering many potential wall geometries without the computational overhead of complex finite element or finite difference numerical models.
\end{abstract}

KEYWORDS retaining walls; design; clays 


\section{INTRODUCTION}

Conventional design of retaining walls in clay is based on plasticity theory in which zones of soil on the active and passive sides of the wall are assumed to reach failure resulting in plastic collapse of the wall. In order to both prevent collapse due to uncertainty in the selection of soil parameters and to limit deformations of surrounding structures, factors of safety are applied, ensuring that soil stresses are far from their values at failure. While arbitrary factors of safety can be rationally used to prevent failure on the basis of statistical variations in soil strength, any design to limit wall deformations should involve the assessment of soil stiffness and strength. In current design practice, the assessment of wall deformation is usually based on elasticity theory using beam on elastic foundation analyses with earth pressures being limited by active and passive values. Finite element analysis is sometimes also used, but rarely with models more complex than elastic perfectly-plastic. The application of elastic analyses to what is fundamentally a non-linear plastic material is often complex and based on the selection of an appropriate elastic modulus for the soil before the strain level is known.

Osman and Bolton (2004) derived a mobilisable strength design procedure for rigid retaining walls in clay, invoking the mechanism of Bolton and Powrie (1988) to calculate an appropriate strain level in the soil next to a rotating wall. They then invoked the assumption of a constant mobilised soil strength with depth in order to calculate the soil stress and hence strain required to satisfy equilibrium. The displacements of the wall could then be calculated based on horizontal moment equilibrium. Osman and Bolton (2006) extended this work to braced excavations by assuming a cosine-shaped wall deformation profile between the lowest support and the base of the retaining wall and balancing the virtual loss of potential energy with the virtual plastic work in shearing. While this analysis technique was shown to approximately 
Wang et al. (2018) modified the mobilisable strength design method (MSD) by implementing a more realistic deformation mechanism consisting of modified incremental wall displacement and ground deformation profiles and hence a modified distribution of shear strain. Figure 1 shows comparisons of the lateral wall displacements measured and those calculated by the modified mobilisable strength design method (MMSD) and conventional MSD when the excavation depth reached $46 \%$ and full planned formation level at the Yishan Station, Shanghai, China. Although the magnitudes and shapes of the lateral wall displacement predicted by MMSD came closer to the measurement than those calculated by MSD, the wall deflection shape is still limited by the user-defined function. The transformation of the wall deflection profile from a cosine to an exponential shape may provide some benefits, but the real deformed shape of the wall is still not well reproduced.

Diakoumi and Powrie (2013) presented a technique for carrying out mobilisable strength design for flexible retaining walls, in which the soil strain was calculated based on the superposition of the mechanisms proposed by Bolton and Powrie (1988) for rotation of each of a series of wall segments. However, the superposition of the four mechanisms utilised and shown in Figure 2 would imply a discontinuous wall shape with the wall shearing instead of bending at each hinge (Haigh et al., 2013). Nevertheless, the analysis provides a useful starting point in working towards a mobilisable strength design procedure for flexible retaining walls that allows equilibrium to be maintained without dictating the deflected shape of the wall. 


\section{PROPOSED ANALYSIS PROCEDURE}

The analysis procedure developed here builds on the work of Diakoumi and Powrie (2013) in assuming that the stress state of soil adjacent to a particular wall segment is a function of the strain mobilised at that point. The strain distribution can be calculated for any given wall shape by assuming a compatible deformation mechanism with the corresponding earth pressures being subsequently calculated through a simplified constitutive model. Finally, iteration allows equilibrium to be achieved between the earth pressures acting upon the wall and the stresses within the wall due to its deflected shape.

Two conversions must be derived before carrying out this analysis procedure. Firstly the soil strains must be associated with the deflected shape of the retaining wall via a deformation mechanism, and secondly the mobilised strength of the soil must be linked to the soil strain via a simplified constitutive model.

\section{Assumed Deformation Mechanism}

To overcome the shortcomings of the analyses of Diakoumi and Powrie (2013), as discussed in Haigh et al. (2013), the wall deformation was calculated by the superposition of a series of hinging mechanisms in which the top and bottom of the retaining wall are pinned, with the wall deflecting as two straight segments connected by a hinge between them. As can be seen from Figure 3, the superposition of a number of these hinging mechanisms together with a rigid wall rotation about the base and a rigid wall translation can allow a complete set of displacement profiles to be evaluated for both simple cantilever walls and those with multiple rigid or flexible props. While only three hinging mechanisms are shown in the figure for clarity, any number of 
these could be superposed to give a larger number of wall elements and hence a more accurate solution.

Bolton and Powrie (1988) proposed a simplified admissible strain field behind a frictionless rigid wall rotating outwards by a small angle about the base, as shown in Figure 4. The horizontal, vertical and shear strain increments in the strain field are assumed to be uniform within the deforming triangular wedge AVO with values given by Equations 1-3, in which positive values are compressive. By assuming constant volume, appropriate for the undrained behaviour of clays, the zero-extension line OA can be shown to be at 45 degrees to the principal directions, which are horizontal and vertical.

$$
\begin{gathered}
\delta \varepsilon_{h}=-\delta \theta \\
\delta \varepsilon_{v}=+\delta \theta \\
\delta \gamma=2 \delta \theta
\end{gathered}
$$

where $\delta \theta$ is the wall rotation angle increment about the base as shown in Figure 4.

Hinging mechanisms can be created by the superposition of two of the mechanisms in Figure 4 as illustrated in Figure 5. The first mechanism is wall rotation about the wall base with an angle of $\frac{x}{L-x} \theta$ outwards. The second mechanism involves the lower portion of the wall remaining vertical with the wall above a hinge at depth $x$ rotating an angle of $\frac{L}{L-x} \theta$ inwards. The summation of these mechanisms gives zero displacement at the wall top with the top section having rotated by an angle of $\theta$. By superposition of the strains calculated from equation 3 , the magnitudes of the shear strains of the soil above and below the hinge are $2 \theta$ and $\frac{2 x}{L-x} \theta$ respectively.

A simplified admissible strain field behind a frictionless rigid wall translating outwards by a small distance was proposed by Bolton and Powrie (1988), who deduced the uniform 
horizontal, vertical and shear strain increments in the rectangle AVOB as shown in Equations 4-6.

$$
\begin{gathered}
\delta \varepsilon_{h}=-\frac{\delta u}{h} \\
\delta \varepsilon_{v}=+\frac{\delta u}{h} \\
\delta \gamma=2 \frac{\delta u}{h}
\end{gathered}
$$

where $\delta u$ is the horizontal translational displacement increment and $h$ is the wall height as shown in Figure 6.

\section{Simplified Constitutive Law}

To assess the stresses applied to the wall once the strains are known, a simple relationship linking the shear stress acting on a soil element to its shear strain is needed. This need not be as sophisticated as a full finite element constitutive model and is essentially just a shear stressshear strain curve. Vardanega and Bolton (2011) presented such a model based on 115 isotropically consolidated triaxial, direct shear and cyclic tests on 19 different clays and silts. They postulated that the shear stress-strain law can be approximated by:

$$
\frac{1}{M}=\frac{\tau_{m o b}}{c_{u}}=0.5\left(\frac{\gamma}{\gamma_{M=2}}\right)^{b} \quad \text { for } 1.25<M<5
$$

where $\tau_{m o b}$ is the mobilised shear stress, $c_{u}$ is the peak undrained shear strength, $\gamma$ is the shear strain, $\gamma_{M=2}$ is the strain when half of the $c_{u}$ is mobilised and $b$ is an experimentally derived exponent, normally taken as 0.6 . The mobilisation factor, $M$, describes the mobilisation of shear strength (BSI, 1994) being equal to the factor of safety for the soil element. The value of $\gamma_{M=2}$ would ideally be measured via a triaxial test but could alternatively be predicted using:

$$
\gamma_{M=2}=0.0109\left(I_{p}\right)^{0.45}\left(\frac{c_{u}}{p_{0}^{\prime}}\right)^{0.59}\left(\frac{p_{0}^{\prime}}{p_{a t m}}\right)^{0.28}
$$


where $I_{p}$ is the plasticity index, $p_{0}^{\prime}$ is the initial mean effective stress and $p_{a t m}$ is the atmospheric pressure with a value of $101.3 \mathrm{kPa}$ (Vardanega and Bolton, 2011).

For very high strains, equation 7 will predict strengths greater than $\mathrm{c}_{\mathrm{u}}$. In the code presented here the mobilised strength is limited to $c_{u}$ for high strains.

\section{IMPLEMENTATION}

The analysis procedure was implemented in MATLAB in order to evaluate the performance of the code in predicting the undrained movements of flexible retaining walls. The software allows the analysis of cantilever walls or walls with any number of props with user-defined prop stiffness and potential lack of fit. A flow chart of the analysis procedure is given in Figure 7.

The wall is discretised into a number of elements, each of which is associated with a hinging mechanism as shown in Figure 3. For a given wall deformation, the strains within the soil in front of and behind the wall are calculated from superposition of the shear strain fields given by equations 3 and 6 . As the shear strains due to rotation and translation act in different regions of soil, as shown in figures 4 and 6, a representative shear strain is calculated using equation 9.

$$
\gamma=\sqrt{\gamma_{\text {rotation }}^{2}+\gamma_{\text {translation }^{2}}}
$$

This representative shear strain is then used to calculate the mobilised shear stress in each element of soil using equation 7 , with horizontal earth pressures being calculated using equation 10. The direction of wall movement relative to the soil is used in the determination of active or passive loading.

$$
\sigma_{h}=\sigma_{v} \pm 2 \tau_{m o b}
$$


The use of equation 10 implies that horizontal stresses are equal to vertical stresses for very low wall deformations, i.e. $K_{0}=1$. While this assumption may be inaccurate in many cases, the modest displacements of even relatively rigid walls are such that this does not dominate the analytical results at least for the cases analysed here. The assumption also matches with the isotropic consolidation condition in the triaxial tests utilised to derive the stress strain law of equation 7. Very stiff prop systems may result in low wall displacements for which this effect may become more important.

Prop loads are calculated based on axial shortening of the props due to horizontal wall deflections. As props may be installed at any point in the construction process, the user inputs the horizontal movement at which zero load is achieved together with the axial stiffness of the prop. This allows slack in the prop at installation to be implemented if necessary, which has been shown to have an important influence on prop loads by Twine and Roscoe (1999). In the procedure described here the props are assumed to be attached in such a way that they only act in compression and not in tension.

These horizontal earth pressures are then numerically integrated twice to calculate the bending moment distribution applied to the wall. The bending moments due to the prop loads are then added. The wall deflections are numerically differentiated twice and multiplied by the wall bending stiffness $E I$ to calculate the bending moment distribution required to maintain the wall shape. The difference between these bending moments is used to calculate a vector of out-ofbalance moments. To eliminate this error term, the wall shape must be iterated based on the tangent stiffness matrix. As the soil behaviour is non-linear, the tangent stiffness matrix is assembled by incrementing each degree of freedom of the wall in turn by a small value and monitoring the change of the bending moment error at each node of the wall. 
After inverting the stiffness matrix and multiplying by the bending moment error to calculate the change in wall shape required, the software iterates to find a solution in which the retaining wall is in equilibrium in its deformed shape under the action of the applied earth pressures and prop forces. The iteration of the wall bending moment causes the bending moment error to drop to a user-defined limit. Whilst this brute-force approach to determining the stiffness matrix is inelegant, the resulting code converges very rapidly without the need to analytically calculate the stiffness matrix. This feature was incorporated into the algorithm in order to cope with experimental stress-strain data which did not have a simple analytical form. While equation 7 is smooth in the range specified, for very high strains it will predict mobilised shear stresses greater than the strength of the soil and must thus be truncated. This discontinuity in the slope of the stress-strain curve makes direct calculation of the stiffness matrix more complex. The process also allows stress-strain data to be input directly without fitting an analytical function.

For walls in which the construction sequence is modelled, for example where props are installed once excavation has progressed to a given level, the process is repeated for each construction stage with the wall position at the end of stage $n$ being used as the starting point for stage $n+1$. Deflections calculated at the end of these stages can also be used to define the deflections at which the props are unstressed.

\section{VALIDATION}

To validate the performance of the code, the behaviour of several retaining walls in published case histories and centrifuge tests was analysed, covering very different soil stiffnesses, from very high OCR stiff clays in Dublin to very soft clays in Oslo. The field case histories were 
chosen as data were available both for the deflections of the wall during construction and also for the stress-strain behaviour of the clay allowing $\gamma_{M=2}$ to be directly determined from triaxial test data rather than picked to give the best fit to the observed behaviour. In all of these analyses a prototype spacing of $0.1 \mathrm{~m}$ between nodes was used.

\section{Singly-Propped Retaining Wall (Long et al., 2012)}

Long et al. (2012) presented several Irish case histories of deep excavations with props in Dublin Boulder Clay. One of these case-histories is that of the Dublin Port Tunnel, in which a $12 \mathrm{~m}$ deep excavation was supported by a $24 \mathrm{~m}$ deep diaphragm wall with a prop $1.5 \mathrm{~m}$ below the surface as shown in Figure 8a.

The diaphragm wall was constructed using conventional techniques with $7 \mathrm{~m}$ long bentonite supported panels using rope-mounted clamshell grabs. The wall thickness was $1.2 \mathrm{~m}$ and the stiffness was calculated to be $4.32 \mathrm{GNm}^{2} / \mathrm{m}$ (Curtis and Doran, 2003). The steel props used for supporting the diaphragm wall had a $1220 \mathrm{~mm}$ outside diameter with a $14.2 \mathrm{~mm}$ wall thickness, corresponding to an axial stiffness of $140 \mathrm{MN} / \mathrm{m}^{2}$. The props spanned the $22 \mathrm{~m}$ width of the excavation and were connected directly to the diaphragm wall using reaction pads at $7 \mathrm{~m}$ centres.

The behaviour of Dublin Boulder Clay was presented by Long and Menkiti (2007), who showed that the heavily over-consolidated upper black and lower brown boulder clays have a very high bulk density of $2.3 \mathrm{Mg} / \mathrm{m}^{3}$. Based on the SPT data presented by Long et al. (2012) as shown in Figure 9, the clay strength was taken to be $120 \mathrm{kPa}$ in the $3 \mathrm{~m}$ thick surface layer of Upper Brown Dublin Boulder Clay and to increase with depth from $138 \mathrm{kPa}$ at $3.5 \mathrm{~m}$ depth to $230 \mathrm{kPa}$ at $7 \mathrm{~m}$ depth in the Upper Black Dublin Boulder Clay. SPT tests were also conducted 
for the Lower Brown and Lower Black Dublin Boulder Clay with the strengths being $376 \mathrm{kPa}$ at $15 \mathrm{~m}$ depth and $409 \mathrm{kPa}$ at $19.5 \mathrm{~m}$ depth respectively. The strength profile was created by interpolating those SPT values and extrapolating them linearly to the depth of the wall toe. Half strength is mobilised at a shear strain of around $0.25 \%$ according to soil stiffness data from high-pressure dilatometer tests and strength data from SPT tests (Long and Menkiti, 2007).

The construction sequence modelled here involved an excavation to a depth of $4 \mathrm{~m}$, followed by installation of steel props at $1.5 \mathrm{~m}$ depth and subsequently continued excavation to a depth of $12 \mathrm{~m}$.

The wall deflections measured using inclinometers and predicted by the method described here are shown in Figure 8b. It can be seen that both the maximum wall deflection and the depth at which it occurred are well characterised by this simple model. It should be noted that inclinometers did not extend to the base of the retaining wall but terminated at a depth of 18 m. This, together with a lack of displacement data to set the integration constant causes a lack of certainty regarding the lateral displacement. It was assumed that the lateral displacement at $18 \mathrm{~m}$ depth was zero when plotting the field data. This assumption is not necessarily true but in the absence of conflicting data is rational.

It can be seen from Figure $8 \mathrm{c}$ that above the excavation level only very small earth pressures develop owing to the high strength of the clay which allows the vertical cut to be almost selfsupporting. Below the excavation level the passive resistance developed is around $30 \%$ of the passive limit value owing to the very low strains mobilised and the high static factor of safety of the wall. The prop load predicted by the model is $1276 \mathrm{kN}$ per prop, compared with $787 \mathrm{kN}$ observed in the field at the moment when the maximum excavation level was reached. This prop load was calculated using the plane-strain equivalent elastic prop stiffness and the wall 


\section{Multi-Propped Retaining Wall (NGI, 1962)}

NGI (1962) presented the results of field measurements at a strutted excavation in soft clay along the Oslo Subway. The excavation comprised a $14.5 \mathrm{~m}$ deep sheet-pile wall with five layers of props supporting a 9.2 m deep excavation as shown in Figure 10.

The steel sheet-pile wall with a stiffness of $61.2 \mathrm{MNm}^{2} / \mathrm{m}$ was driven to bedrock at the beginning of excavation so that the wall toe can be regarded as fixed during the whole process. In the analysis procedure a rigid prop was installed at this location. Steel struts with an axial stiffness of $256 \mathrm{MN} / \mathrm{m}^{2}$ were installed at the first, fourth and fifth layers while another type with an axial stiffness of $130 \mathrm{MN} / \mathrm{m}^{2}$ were used as the second and third layers of props. The five layers of props spanned the $11 \mathrm{~m}$ width of the excavation and the distance between two struts in the same layer was $3.2 \mathrm{~m}$. In the analysis these prop stiffnesses were converted to an equivalent plane-strain value based on the prop spacing.

The stratigraphy comprised $2 \mathrm{~m}$ fill above the level of the retaining wall crest, providing a surcharge of approximately $40 \mathrm{kPa}$, overlying a $14.5 \mathrm{~m}$ thick clay layer with a bulk density of 2.0 Mg/m³ . Based on in situ vane test data presented by NGI (1962), the undrained shear strength of the clay was approximately $23 \mathrm{kPa}$ in the top $2 \mathrm{~m}$ layer increasing to $30 \mathrm{kPa}$ at a depth of $9 \mathrm{~m}$ (excluding the fill layer), below which it stayed constant to the bedrock level. Between the two depths where vane tests were conducted, linear interpolation was used to 


\begin{tabular}{|c|c|c|c|c|c|c|}
\hline Day No. & 3 & 14 & 27 & 46 & 56 & 74 \\
\hline Excavation Depth (m) & 1 & 2.7 & 3.1 & 6.2 & 7.2 & 9.2 \\
\hline Propped Layers & Cantilever & I & I, II & I, II, III & I, II, III, IV & I, II, III, IV,V \\
\hline
\end{tabular}

Figure 11a displays the sheet-pile wall deflections observed using electrical inclinometers and calculated by the numerical model described here. It can be concluded that the deflection during the cantilever stage is well predicted in terms of both the magnitude and the deformed shape. With the increasing excavation and the installation of steel props, the deformed shape of the retaining wall changed gradually and the location where the maximum lateral displacement occurred moved progressively deeper. The deflections on days 14 and 27 are over-predicted 
Table 2. Comparison of observed and predicted summation of strut load

\begin{tabular}{|c|c|c|c|c|c|}
\hline Day No. & 14 & 27 & 46 & 56 & 74 \\
\hline Observed Value (kN) & 452.24 & 530.72 & 1643.18 & 2172.92 & 2812.52 \\
\hline Predicted Value (kN) & 590.39 & 736.22 & 1949.88 & 2322.13 & 2882.45 \\
\hline Error & $31 \%$ & $39 \%$ & $19 \%$ & $7 \%$ & $2 \%$ \\
\hline
\end{tabular}

NGI (1962) recorded a significant change in the construction sequence on day 57. It was thought that the sheet-pile wall was sliding along the bedrock surface due to misinterpretation of the measured sheet-pile movements. To alleviate the so called slide, a series of procedures were conducted including adding clay to the excavation bottom to act as ballast with the excavation level rising from $7.2 \mathrm{~m}$ on day 57 to $5.4 \mathrm{~m}$ on day 60 . The concrete base slab was subsequently completed during the period from days 61 to 67 at the left side of the excavation, 
which was around $10 \mathrm{~m}$ away from the observed location (Pile 12a). The construction returned to Pile $12 \mathrm{a}$ with excavation to a depth of $8.1 \mathrm{~m}$ and the fifth layer of props was installed on day 71 , followed by the final excavation to a depth of $9.2 \mathrm{~m}$ on day 74 .

This unplanned but flexible operation of re-filling was also modelled by the numerical code through assigning the refilling level of $5.4 \mathrm{~m}$ as a new excavation depth and keeping all the existing props, the iteration would start from the deformed wall in last excavation stage and end up with a negative deflection increment under a new equilibrium of bending moment. Reexcavation was conducted and followed by the installation of the fifth layer of props, which were modelled by the code as they were before. It can be observed in Figure 11a that the predicted deflection matches well with that observed in terms of the magnitude and the shape, which is also validated through the comparison of the summation of strut load as shown in Table 2.

\section{Centrifuge Modelled Multi-Propped Retaining Wall (Xu, 2018)}

One centrifuge test was conducted at $85 \mathrm{~g}$ to simulate a $12.8 \mathrm{~m}$ deep excavation supported by a $22.1 \mathrm{~m}$ deep flexible retaining wall and three layers of props in the single basket beam centrifuge at Tongji University (Ma et al., 2006).

\footnotetext{
The aluminium model wall simulated a reinforced concrete diaphragm wall with a prototype thickness of $0.9 \mathrm{~m}$ and a stiffness of $1.68 \mathrm{GNm}^{2} / \mathrm{m}$. Ten strain gauge bridges were equally spaced on the front and back sides of the retaining wall to measure the bending moment distribution in the flexible wall and the wall deflection was subsequently deduced by integration.
} 
The retaining wall was supported by three layers of props with prototype depths of $2.1 \mathrm{~m}, 6.4$ $\mathrm{m}$ and $10.6 \mathrm{~m}$ below the surface. Each layer of props offered an axial stiffness of $18.44 \mathrm{MN} / \mathrm{m}^{2}$.

The clay used in this centrifuge test was remoulded Shanghai Clay with a maximum preconsolidation pressure of $622.2 \mathrm{kPa}$. The remoulded Shanghai clay had a bulk density of 1.73 Mg/m³ , comparable to the characteristic grey silt clay in the fourth layer of Shanghai Clay. To measure the undrained strength of the remoulded Shanghai Clay, a piezocone penetration test (CPTU) was conducted far away from the retaining wall after the centrifuge swung down. The undrained shear strength was calculated through the tip resistance and pore water pressure and is shown in Figure 12a, the result was smaller than that might be expected due to the fact that the CPTU was carried out after the centrifuge stopped.

The relationship between the mobilisation of undrained shear strength and shear strain of Shanghai Clay is shown in Figure 13, in which half strength is mobilised at a shear strain of around $0.68 \%$ (Lam and Bolton, 2011). Bolton et al. (2014) conducted a sequence of isotropically consolidated undrained triaxial compression tests on samples cored from intact block samples taken from Shanghai Clay at $8 \mathrm{~m}$ depth and calculated $\gamma_{M=2}$ as $0.78 \%$ and $b$ as 0.448 when the confining pressure was $200 \mathrm{kPa}$. This $b$ value was used in the numerical simulation described here.

The technique developed by Haigh et al. (2010) and Lam et al. (2012) utilising a T-shaped scraper controlled by a two-axis servo actuator was employed to excavate the soil in the centrifuge test with props being sequentially installed. The in-flight excavator is described in detail by $\mathrm{Ma}$ and $\mathrm{Xu}$ (2018). A schematic diagram of the model package is shown in Figure 14, in which half of the model was prepared about the centreline of symmetry. Three layers of 
props were installed in the container to initially support the temporary segments, extending to support the retaining wall during the excavation.

The retaining wall was installed in a slot cut in the clay prior to flight with the crest being at the same level as the clay surface before the centrifuge swung up. At $85 \mathrm{~g}$, the excavation and prop installation were conducted according to the modelling sequence shown in Figure 15. The T-shaped scraper excavated clay in $0.17 \mathrm{~m}$ layers with props being installed as the excavation passed the prop depths. The scraper was able to continue to excavate below the installed props due to its inverted $\mathrm{T}$ shape.

The predicted wall deflections, together with the deflection values deduced from the measured bending moments, are shown in Figure 16a. Figure 16b shows the distribution of earth pressures predicted in the final configuration. It can be seen that the earth pressures on the retained side are close to the active limit at all depths with the exception of the surface of the model at which the top layer of props has pushed the wall back into the retained soil causing a passive pressure bulb to form. On the excavation side, earth pressures approach the passive limit with the exception of the surface at which strains are not yet great enough to fully mobilise the passive resistance. These earth pressures reflect the low factor of safety of the wall which leads to high mobilisation of strength being necessary to ensure stability. The soft Shanghai clay thus experiences high strains and hence high wall displacements are observed.

It can be seen that the predicted deflections during excavation with props are higher than those observed and the predicted depths at which the maximum deflections occurred are not precise. One reason for this may be over-installation of the props. The second layer of props (and potentially others) did not stop instantly when touching the retaining wall, which was 
consequently pushed back some distance, leading to a substantial prop force immediately after installation. This will substantially reduce the wall deflections observed.

In order to simulate this numerically, the second layer of props was installed to achieve a wall displacement at the prop level equal to that at the end of the cantilever stage, as was observed in the experiment. As shown in Figure 16a, the magnitudes of predicted wall deflections were closer to those observed when the prop installation was faithfully modelled and the deformed shape of the wall in the last excavation stage was characterised very well. Although this centrifuge test was not perfect due to the inaccurate measurement of the remoulded Shanghai Clay strength and the excessive installation of the second layer of props, it was still a good validation of the code for designing flexible retaining walls in clay.

\section{CONCLUSIONS}

Conventional design of retaining walls in clay based on ultimate limit state analysis can require large arbitrary factors of safety to limit wall deformations. The uncertainty in this process can result in excessively stiff walls being constructed even when some deformation can be tolerated, leading to excessive cost. This paper has presented a very simple numerical method for directly predicting the displacements of propped and un-propped retaining walls during the construction process based on a very simple soil constitutive model which can be calibrated by means of a power-law fitting to conventional triaxial test data.

The simple model has been shown to achieve good accuracy in predicting the observed behaviour of retaining walls in the field in terms of deflections and structural loads with varying prop conditions in both soft and stiff clays. 
This method can give an extremely rapid prediction of retaining wall behaviour during the design process, as setting up the model only requires command-line input of geometric and stiffness parameters and each stage of excavation converges in around $10 \mathrm{~s}$. This method thus allows designers to rapidly assess the performance of different wall geometries and construction sequences providing an extremely valuable tool for propped wall design.

\section{REFERENCES}

Bolton, M. \& Powrie, W. (1988). Behaviour of diaphragm walls in clay prior to collapse. Géotechnique 38, No. 2, 167-189, https://doi.org/10.1680/geot.1988.38.2.167.

Bolton, M. D., Lam, S.-Y., Vardanega, P. J., Ng, C. W. \& Ma, X. (2014). Ground movements due to deep excavations in Shanghai: Design charts. Frontiers of Structural and Civil Engineering 8 , No. 3, 201-236.

BSI, B. S. I. (1994). Code of Practice for Earth Retaining Structures: BS 8002. BSI, London, UK.

Curtis, P. \& Doran, J. (2003). Retaining wall behaviour at the Dublin Port Tunnel. BE Project Civil Engineering Department, University College Dublin, Ireland.

Diakoumi, M. \& Powrie, W. (2013). Mobilisable strength design for flexible embedded retaining walls. Géotechnique 63, No. 2, 95-106, https://doi.org/10.1680/geot.11.P.044.

Haigh, S. K., Houghton, N. E., Lam, S. Y., Li, Z. \& Wallbridge, P. J. (2010). Development of a 2D servoactuator for novel centrifuge modelling. Proceedings of the 7th International Conference on Physical Modelling in Geotechnics, Zurich, Switzerland (eds S. Springman, J. Laue and L. Seward), pp. 239-244. Boca Raton, Florida, USA: CRC Press.

Haigh, S. K., Diakoumi, M. \& Powrie, W. (2013). DISCUSSION: Mobilisable strength design for flexible embedded retaining walls. Géotechnique 63, No. 12, 1080-1082, https://doi.org/10.1680/geot.13.D.01.

Lam, S. \& Bolton, M. (2011). Energy conservation as a principle underlying mobilizable strength design for deep excavations. Journal of Geotechnical and Geoenvironmental Engineering 137, No. 11, 1062-1074.

Lam, S. Y., Elshafie, M. Z. E. B., Haigh, S. K. \& Bolton, M. D. (2012). A new apparatus for modelling excavations. International Journal of Physical Modelling in Geotechnics 12, No. 1, 24-38.

Long, M., Brangan, C., Menkiti, C., Looby, M. \& Casey, P. (2012). Retaining walls in Dublin Boulder Clay, Ireland. Proceedings of the Institution of Civil Engineers - Geotechnical Engineering 165, No. 4, 247-266.

Long, M. \& Menkiti, C. O. (2007). Geotechnical properties of Dublin Boulder Clay. Géotechnique 57, No. 7, 595-611, https://doi.org/10.1680/geot.2007.57.7.595.

Ma, X., He, Z., Zhu, H. \& Lin, M. (2006). Development of a new geotechnical centrifuge at Tongji University in Shanghai. Proceedings of the 6th International Conference on Physical Modelling in Geotechnics, pp. 151-156, Hongkong, China, CRC Press.

Ma, X. \& Xu, J. (2018). Centrifuge model tests on excavation in Shanghai clay using in-flight excavation tools. Proceedings of the 9th International Conference on Physical Modelling in Geotechnics, pp. 419-423, London, United Kingdom, CRC Press.

Norwegian Geotechnical Institute. (1962). Measurements at a strutted excavation, Oslo Subway, Vaterland 1, km 1373. NGI Technical Report. No. 6, Oslo. 
Osman, A. S. \& Bolton, M. D. (2004). A new design method for retaining walls in clay. Canadian Geotechnical Journal 41, No. 3, 451-466.

Osman, A. S. \& Bolton, M. D. (2006). Ground movement predictions for braced excavations in undrained clay. Journal of geotechnical and geoenvironmental engineering 132, No. 4, 465477.

Twine, D. \& Roscoe, H. (1999). Temporary propping of deep excavations: Guidance on design, C517. London, UK: Construction Industry Research and Information Association.

Vardanega, P. \& Bolton, M. (2011). Strength mobilization in clays and silts. Canadian Geotechnical Journal 48, No. 10, 1485-1503.

Wang, L., Liu, Y., Hong, Y. \& Liu, S. (2018). Predicting deformation of multipropped excavations in soft clay with a modified mobilizable strength design (MMSD) method. Computers and Geotechnics 104, No. 12, 54-68.

Xu, J. (2018). Centrifuge modeling of deep retained excavation using inflight excavation tools. Master thesis, Tongji University, Shanghai, China. 


\section{Caption list}

Figure 1. Comparisons of the lateral wall displacements measured and calculated by MMSD and MSD (adapted from Wang et al. (2018))

Figure 2. Superposition of the displaced wall shapes assumed by Diakoumi and Powrie (2013)

Figure 3. Superposition of the wall deformed shapes (Haigh et al 2013)

Figure 4. Compatible mechanism for a frictionless rigid wall rotating about the base (Bolton \& Powrie, 1988)

Figure 5. Admissible strain field for a hinged wall (Haigh et al 2013)

Figure 6. Compatible mechanism for a frictionless rigid wall translation (Bolton \& Powrie, 1988)

Figure 7. Diagram of the calculation procedure in the numerical code

Figure 8. Dublin Port Tunnel (a) excavation layout; (b) comparison of observed and predicted deflections; (c) earth pressures predicted by the model

Figure 9. Ground conditions in Dublin Port Tunnel

Figure 10. Layout of the excavation in Oslo

Figure 11. (a) Deflections observed and predicted of a multi-propped retaining wall in Oslo; (b) Earth pressures predicted at the end of excavation

Figure 12. Excavation in centrifuge test (a) CPTU results for remoulded Shanghai Clay; (b) excavation layout

Figure 13. Mobilisation of undrained shear strength with shear strain of Shanghai Clay (Lam and Bolton, 2011)

Figure 14. Schematic diagram of experimental setup with an in-flight excavator

Figure 15. Modelling sequence of excavation and prop installation

Figure 16. (a) Deflections observed and predicted of a multi-propped retaining wall in the centrifuge test; (b) Earth pressures predicted at the end of excavation

Table 1. Description of the excavation on the days when inclinometer reading was recorded

Table 2. Comparison of observed and predicted summation of strut load 


\section{Notation}

$\delta \varepsilon_{h}$ : the horizontal strain increment of soil

$\delta \varepsilon_{v}$ : the vertical strain increment of soil

$\delta \gamma$ : the shear strain increment of soil

$M$ : the mobilisation factor of undrained shear strength of clay

$\tau_{m o b}:$ the mobilised shear stress

$c_{u}$ : the peak undrained shear strength of clay

$\gamma:$ the shear strain

$\gamma_{M=2}$ : the strain when half of the peak shear strength is mobilised

$b$ : an experimental exponent in the shear stress-strain law, normally taken as 0.6

$I_{p}:$ the plasticity index

$p_{0}^{\prime}$ : the initial mean effective stress

$p_{\text {atm }}$ : the atmospheric pressure

$\gamma_{\text {rotation }}:$ the shear strain due to wall rotation

$\gamma_{\text {translation }}:$ the shear strain due to wall translation

$\sigma_{h}:$ the horizontal stress

$\sigma_{v}:$ the vertical stress

$K_{0}$ : the earth pressure coefficient

$F_{\text {prop: }}$ the prop force 
Normalized lateral wall displacement, $\delta_{\mathrm{h}} / H_{\mathrm{e}}(\%)$

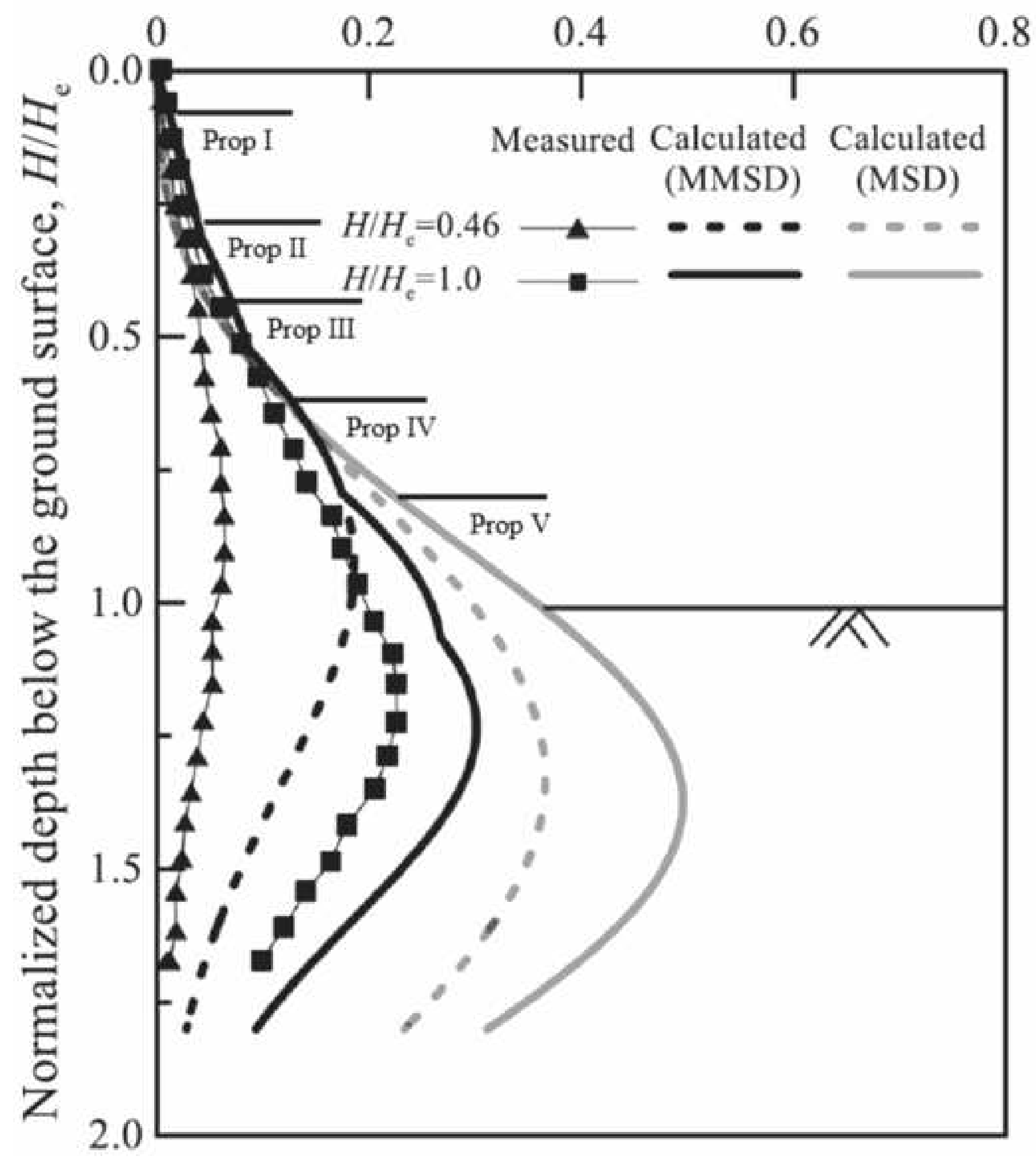




$$
1+4
$$




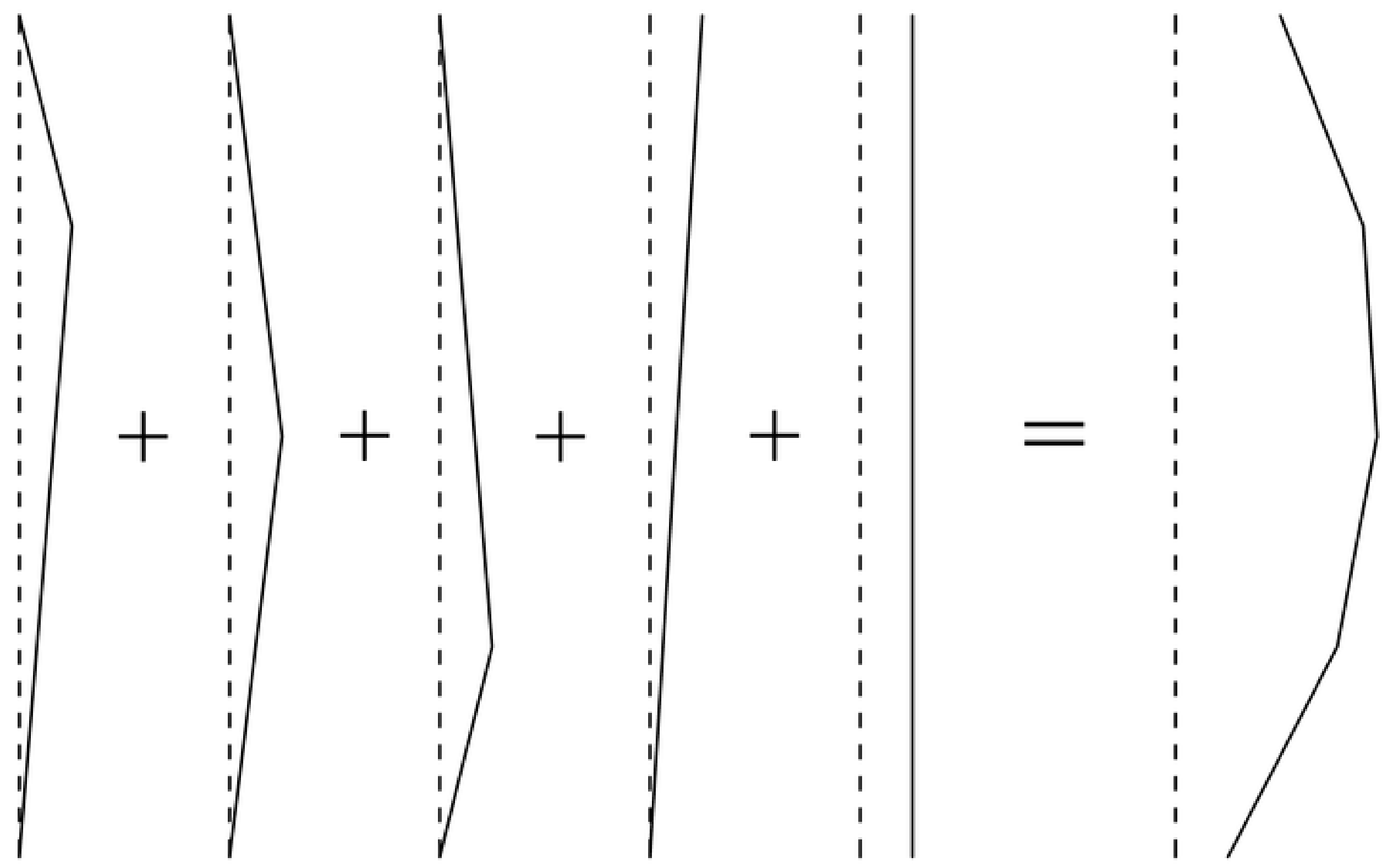

Hinging mechanisms Rotation about base Translation

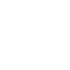



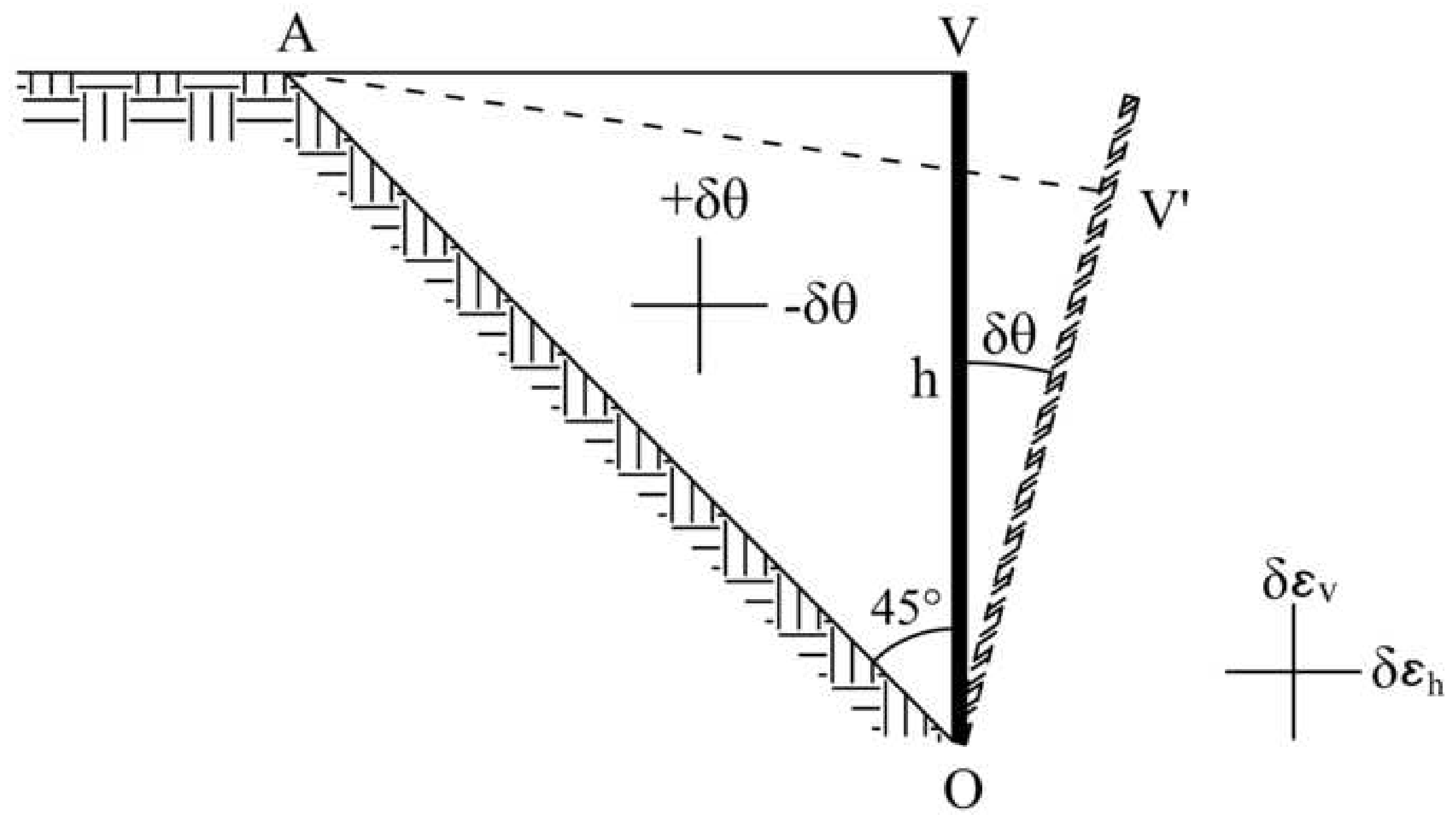

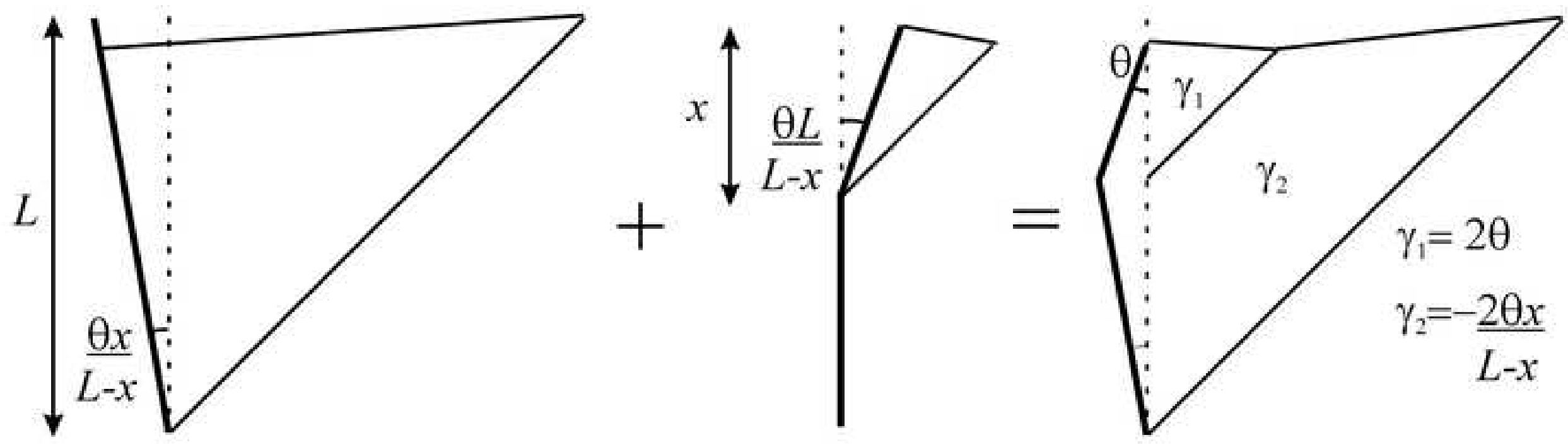


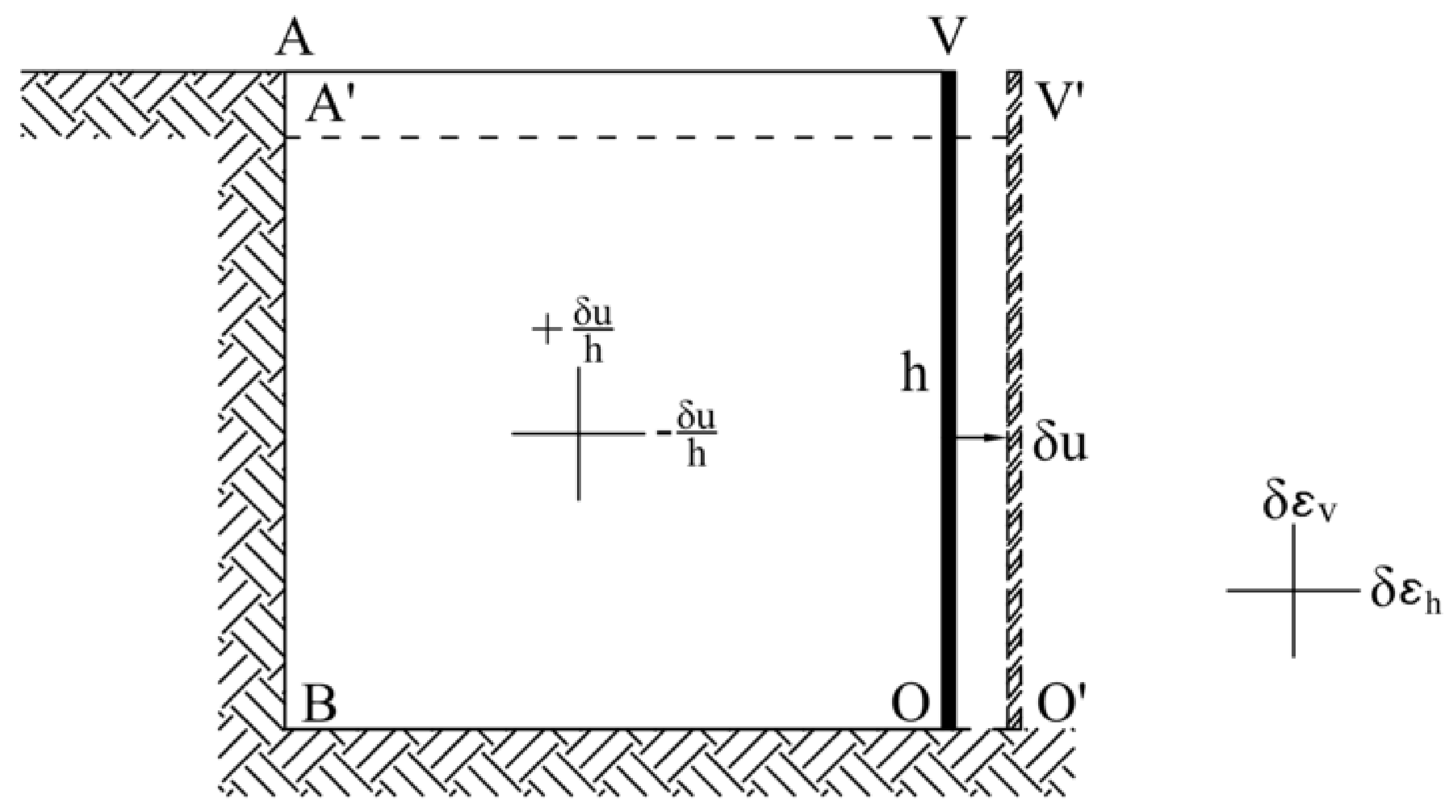




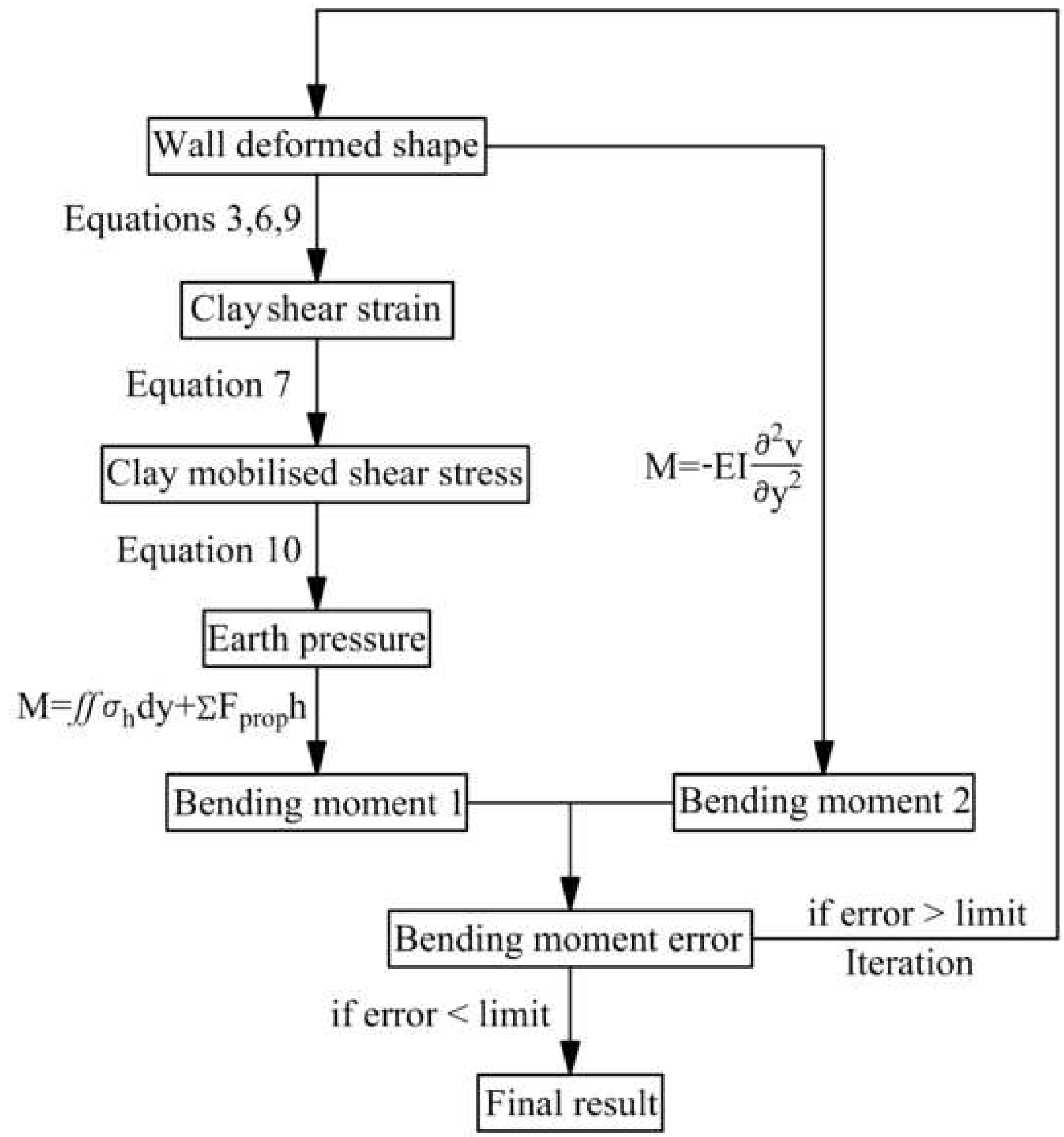




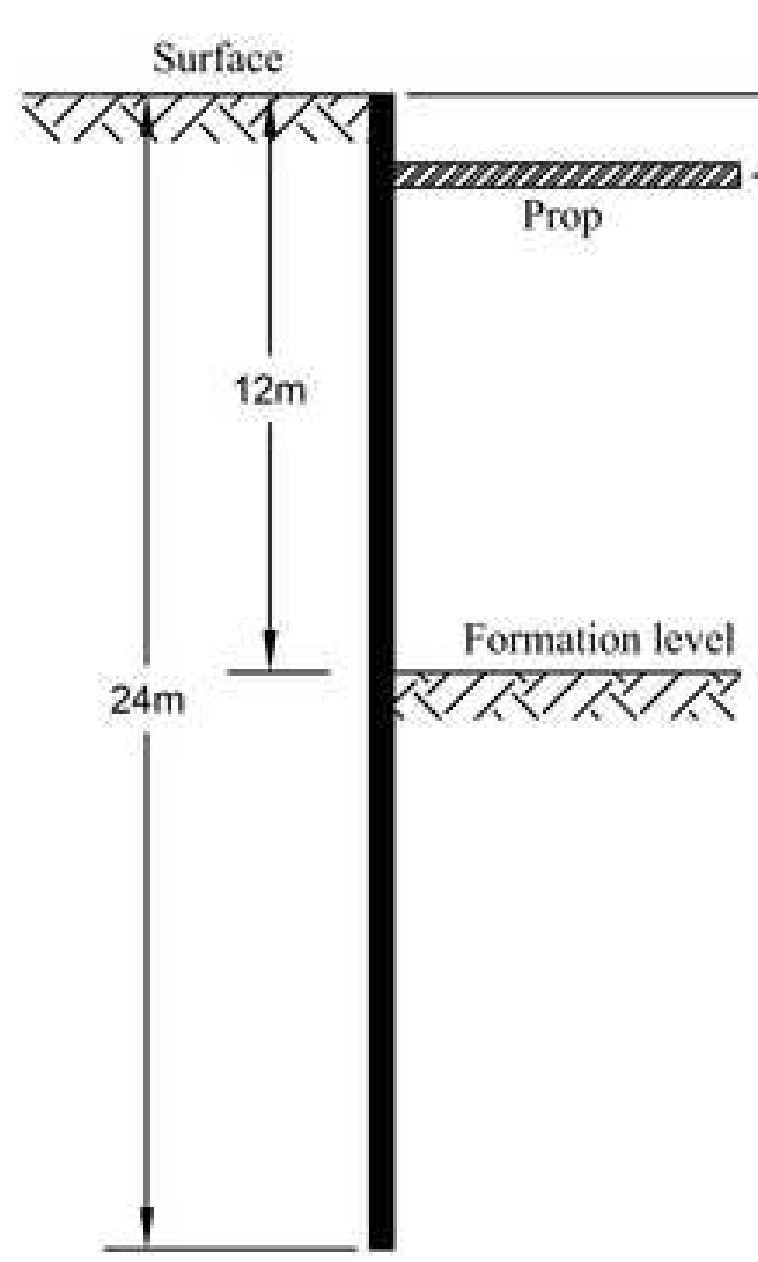

(a)

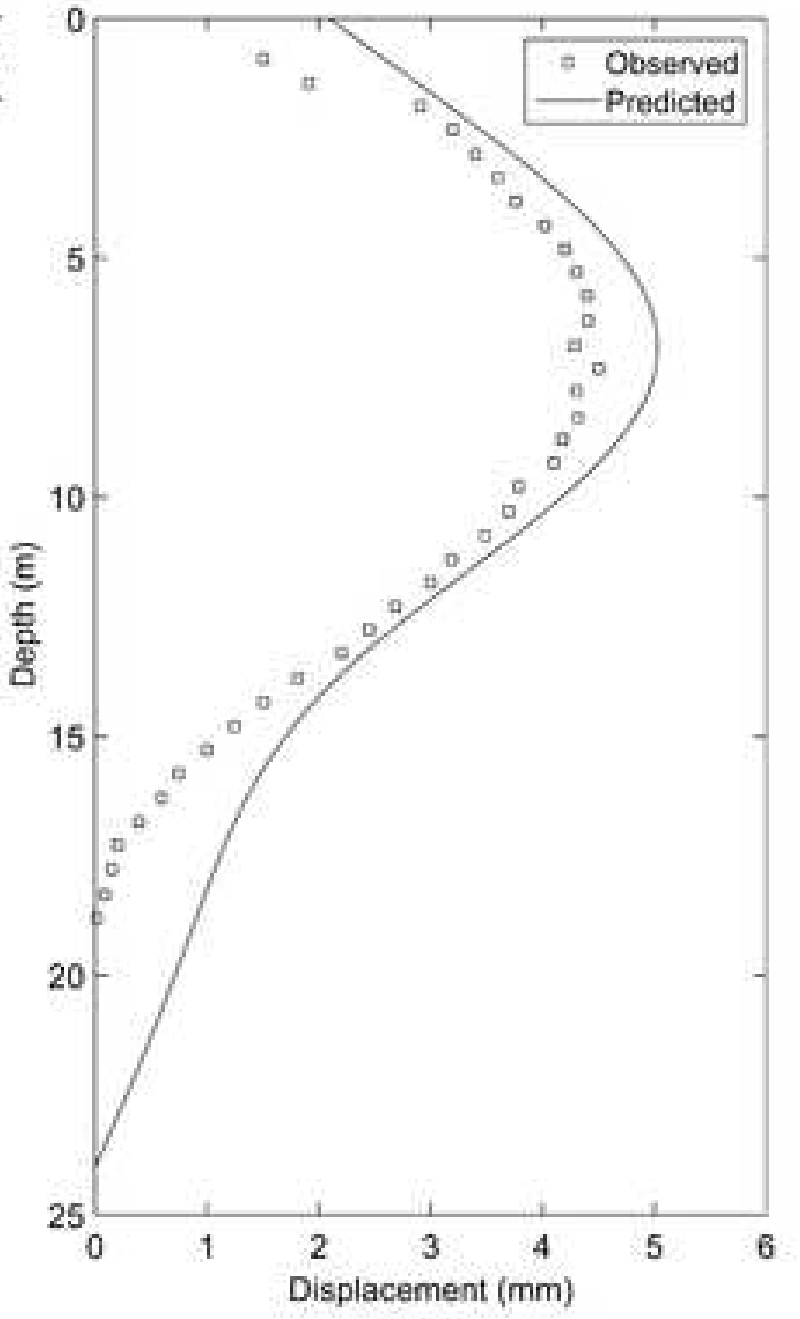

(b)

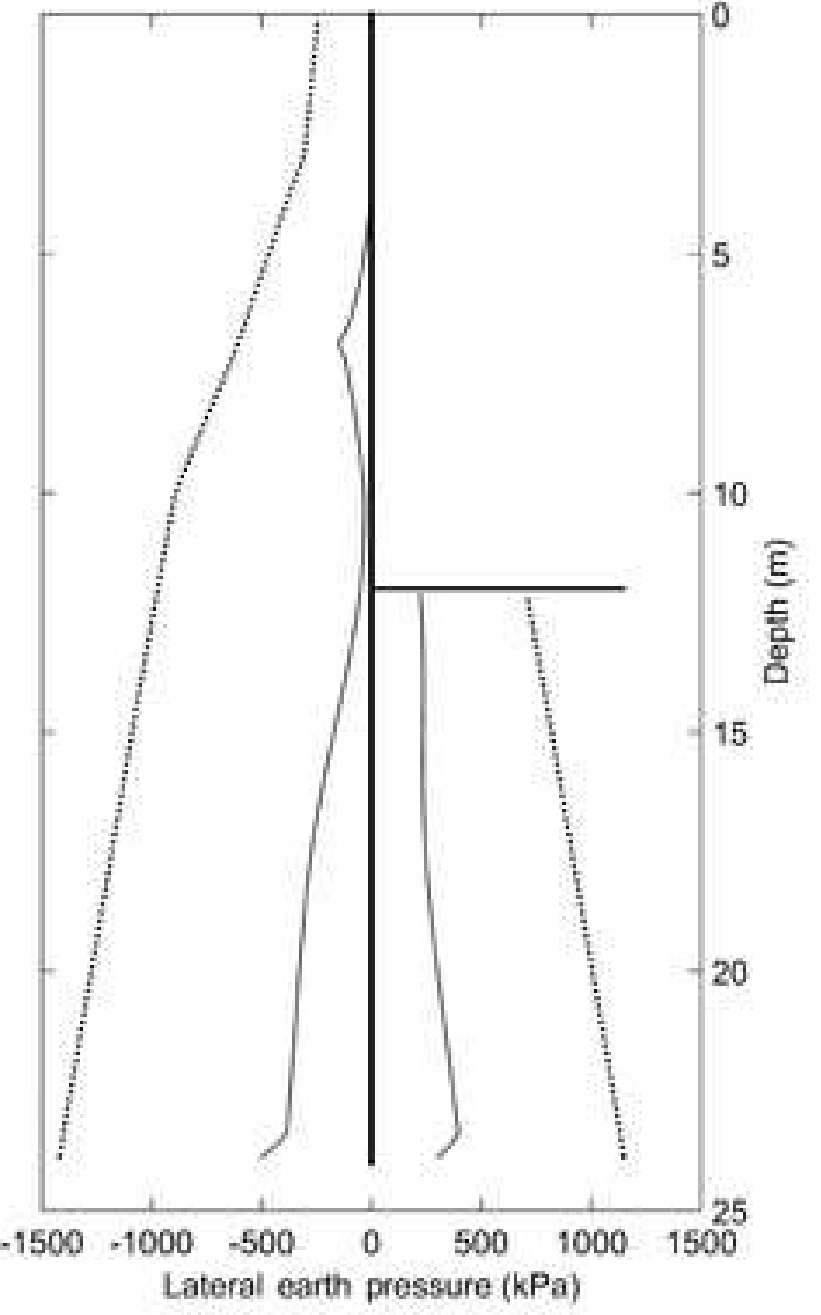

(c) 


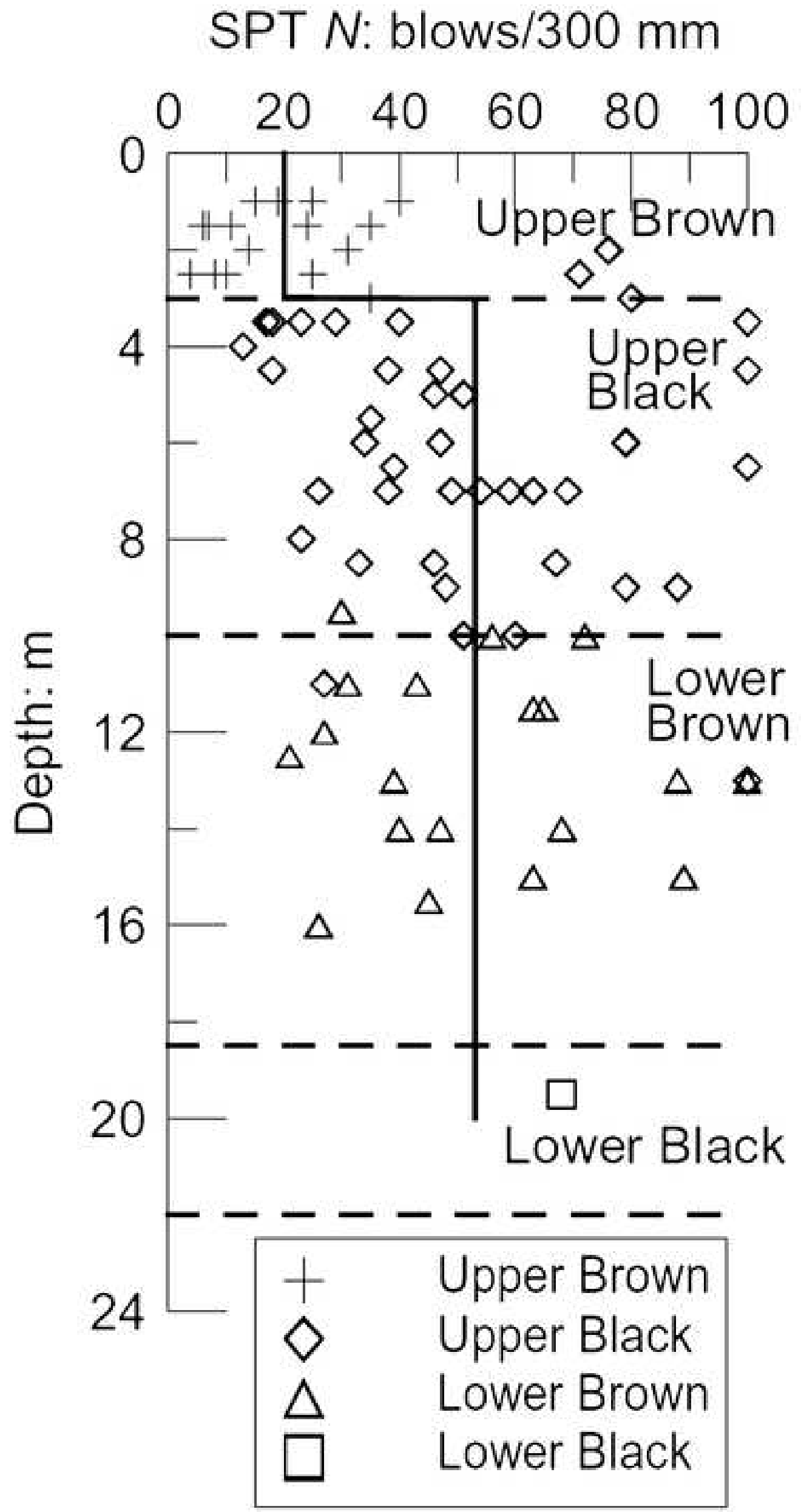




\section{Fill}

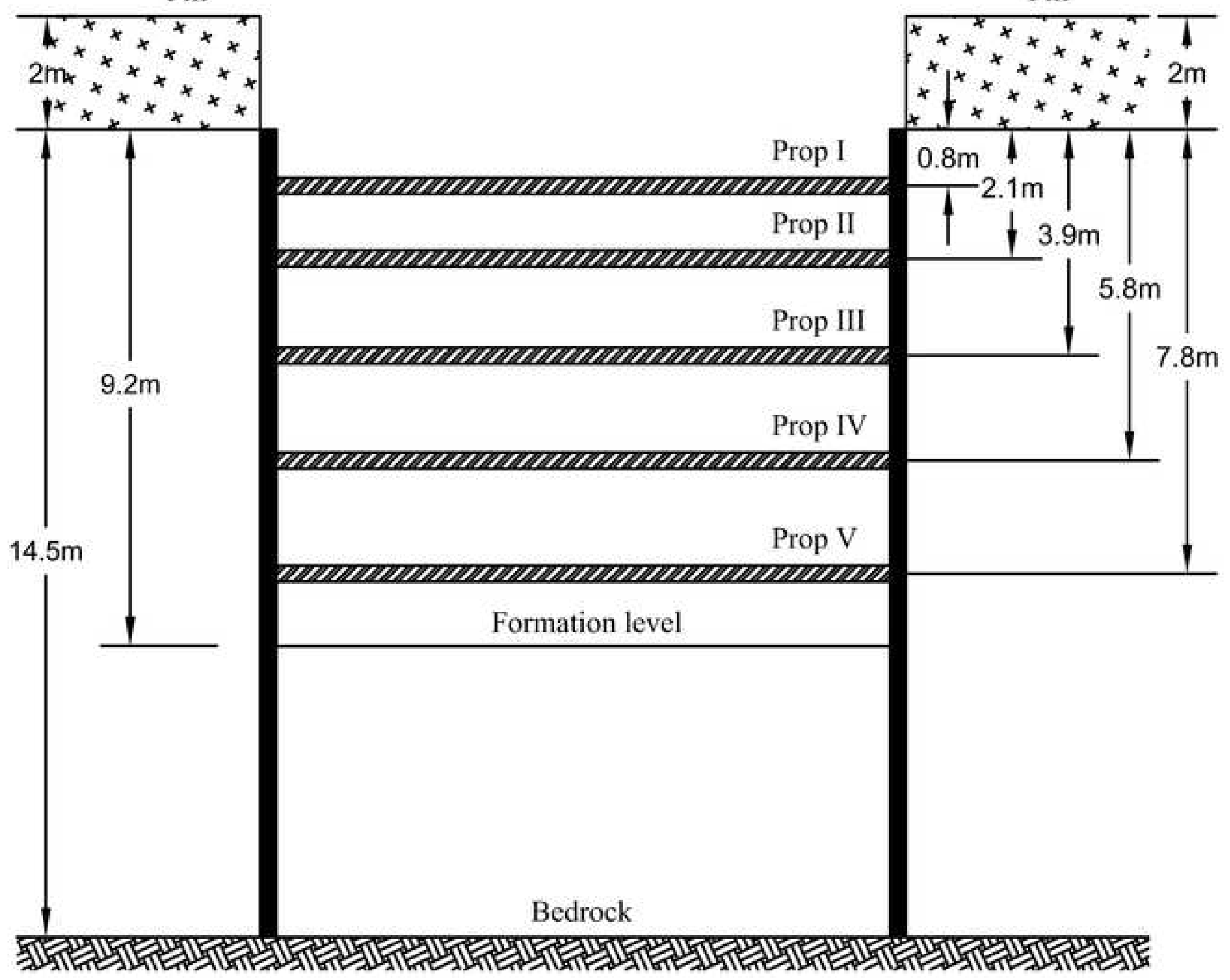



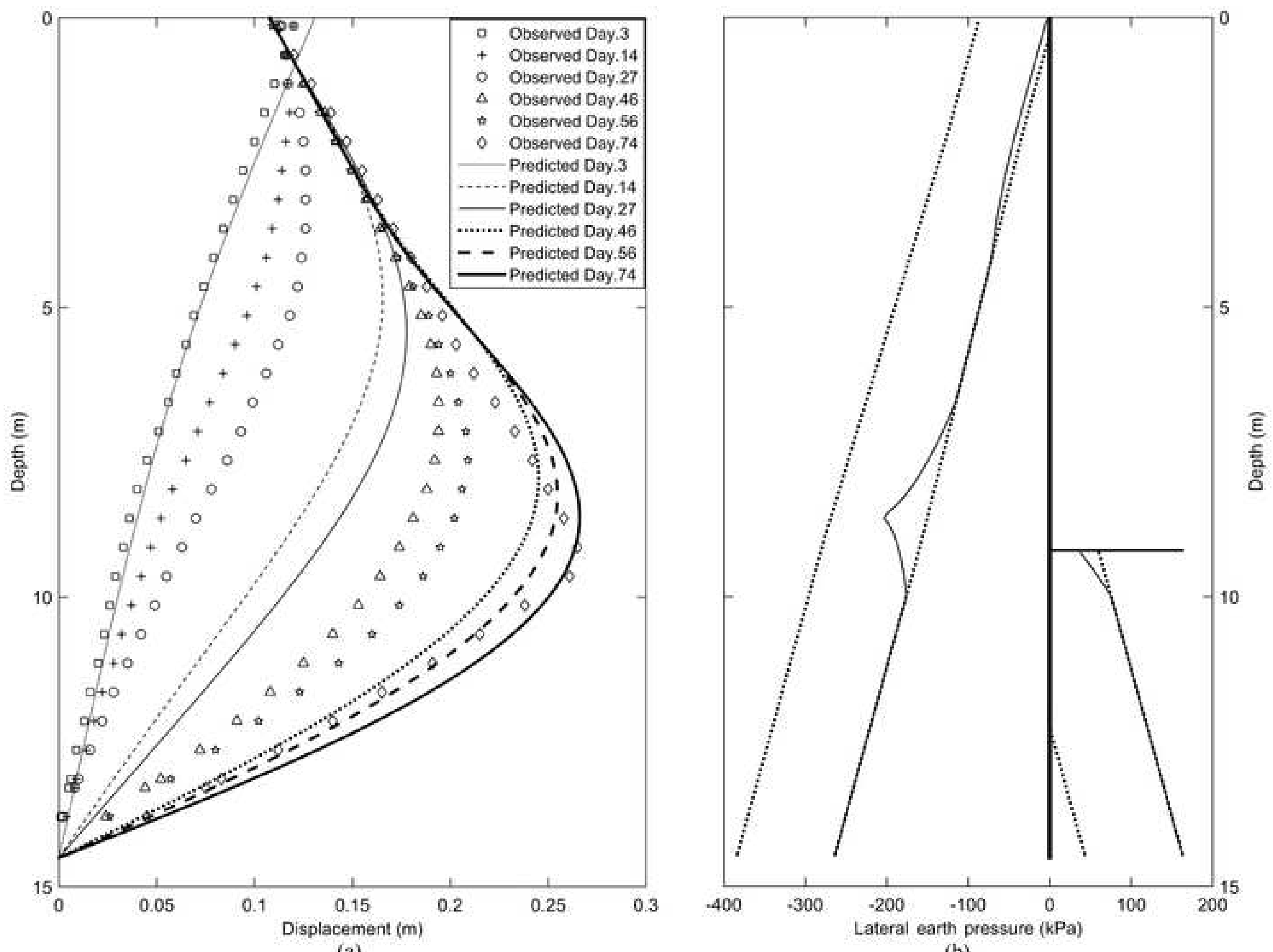

(b) 

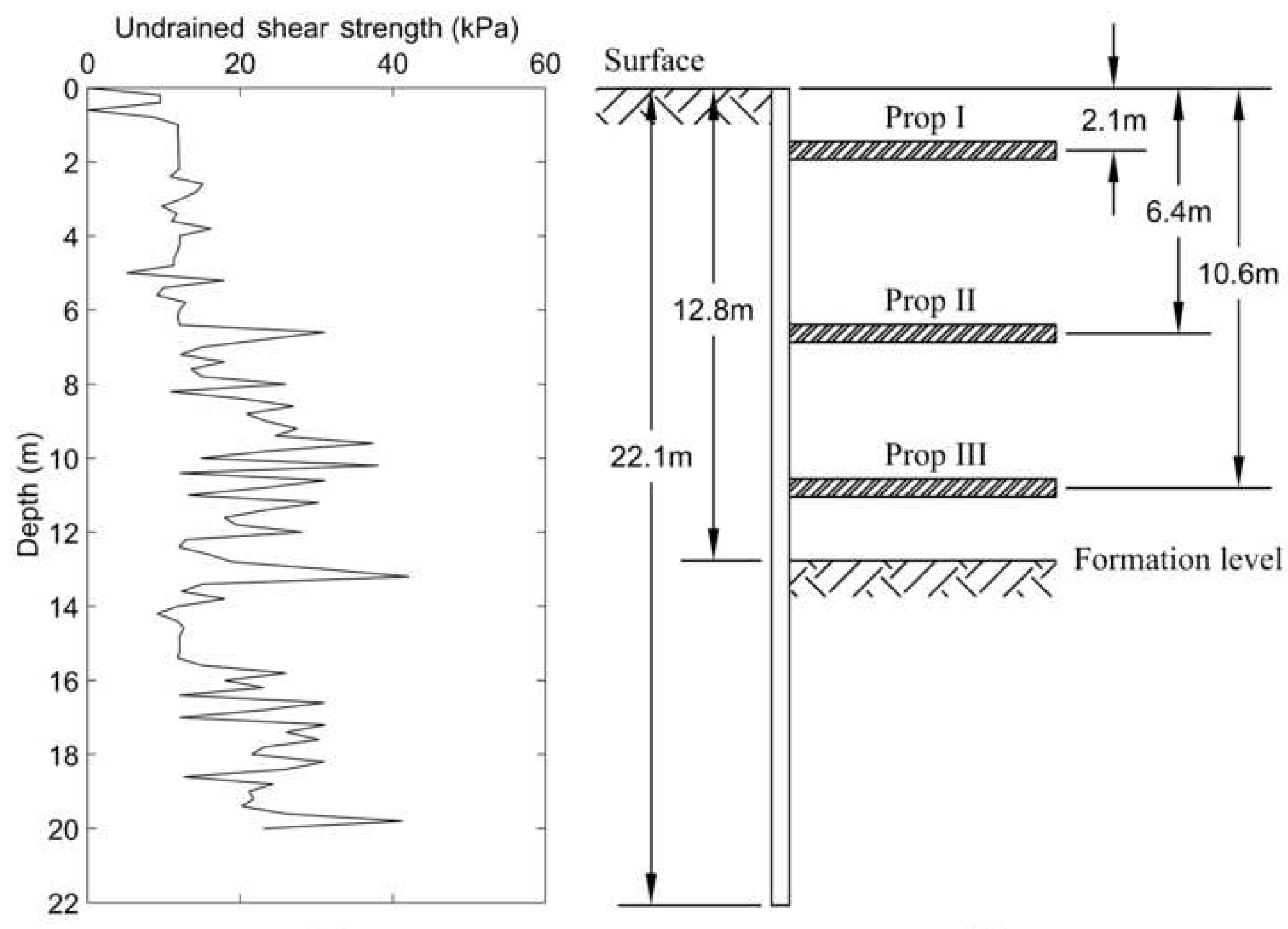

(a)

(b) 


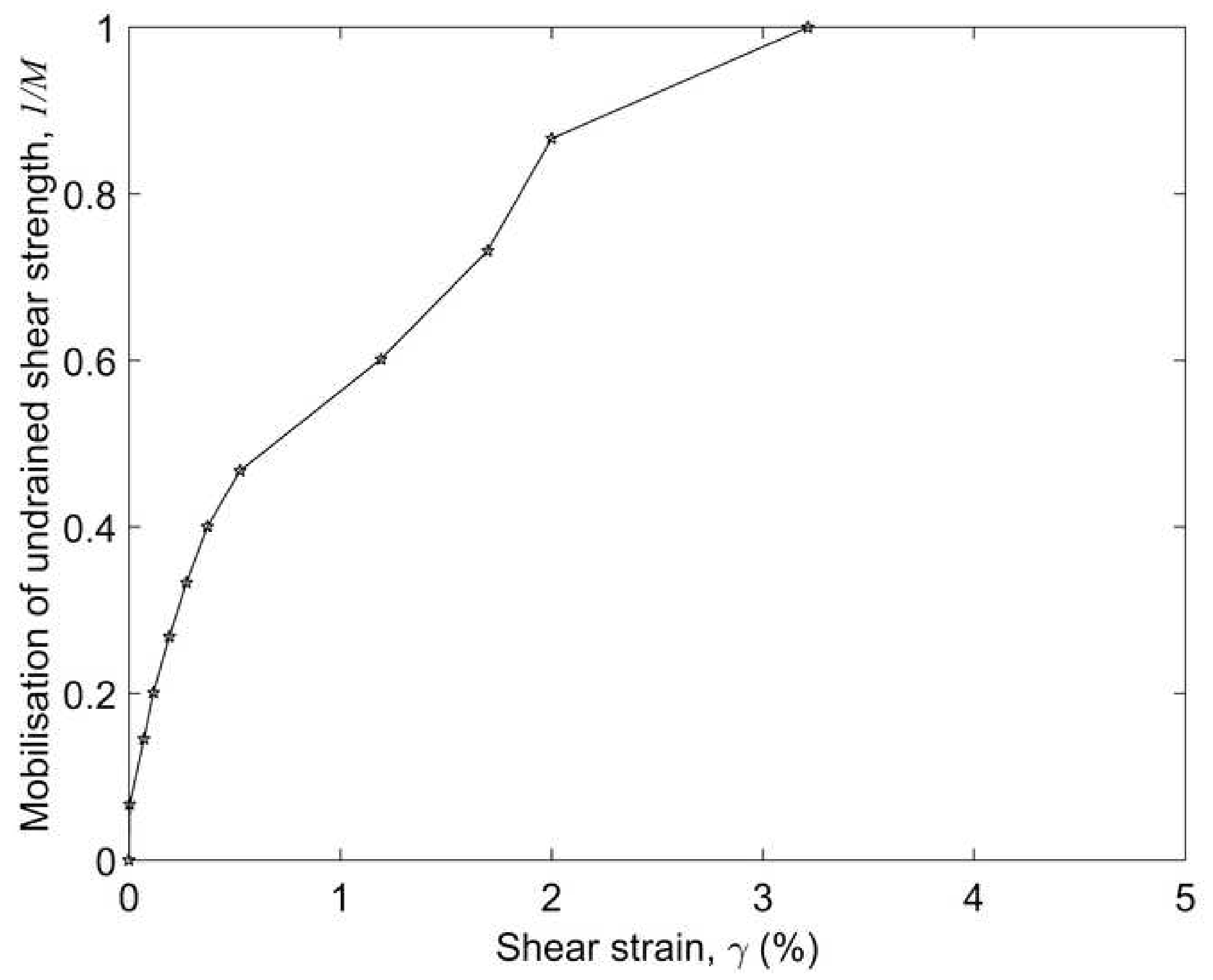




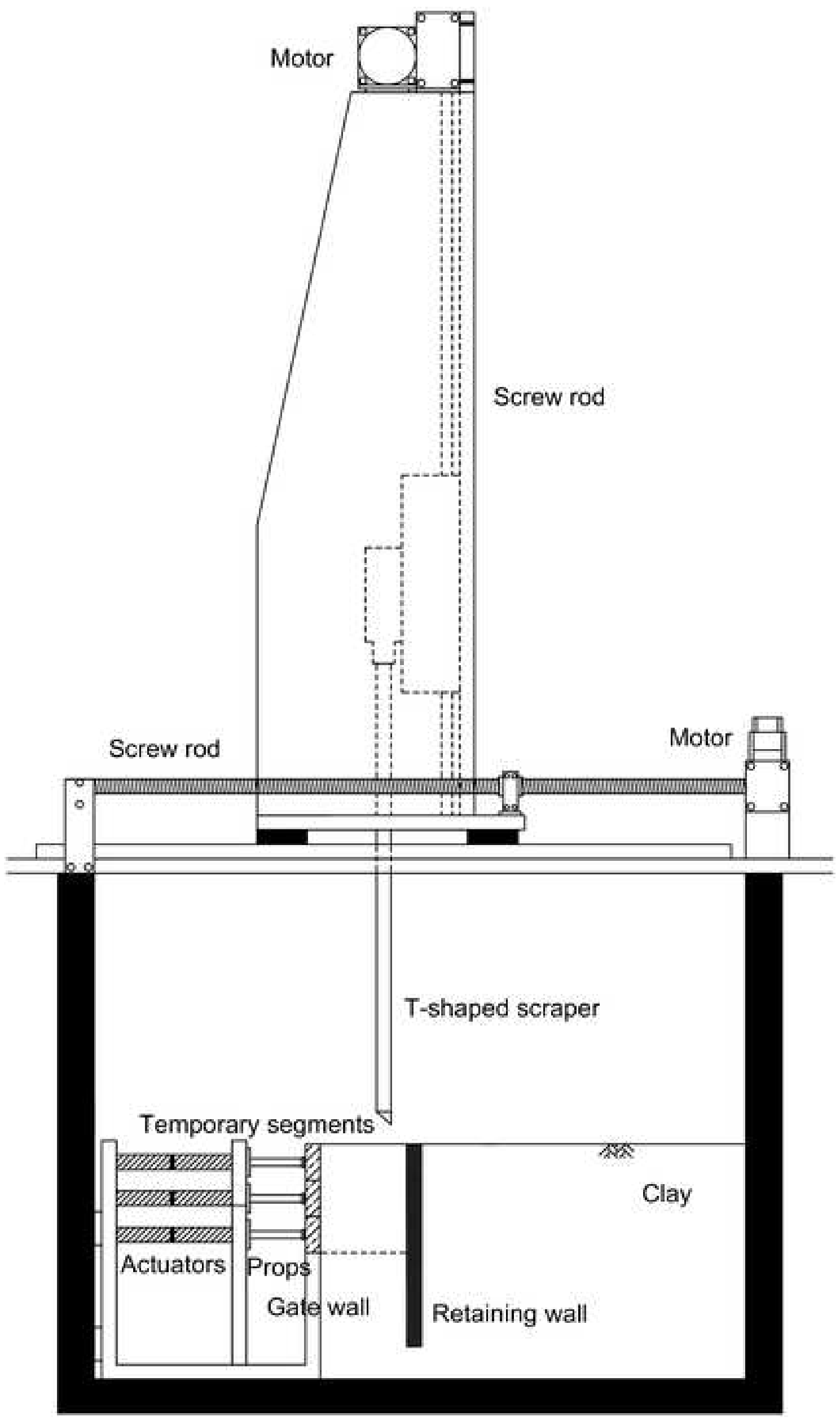




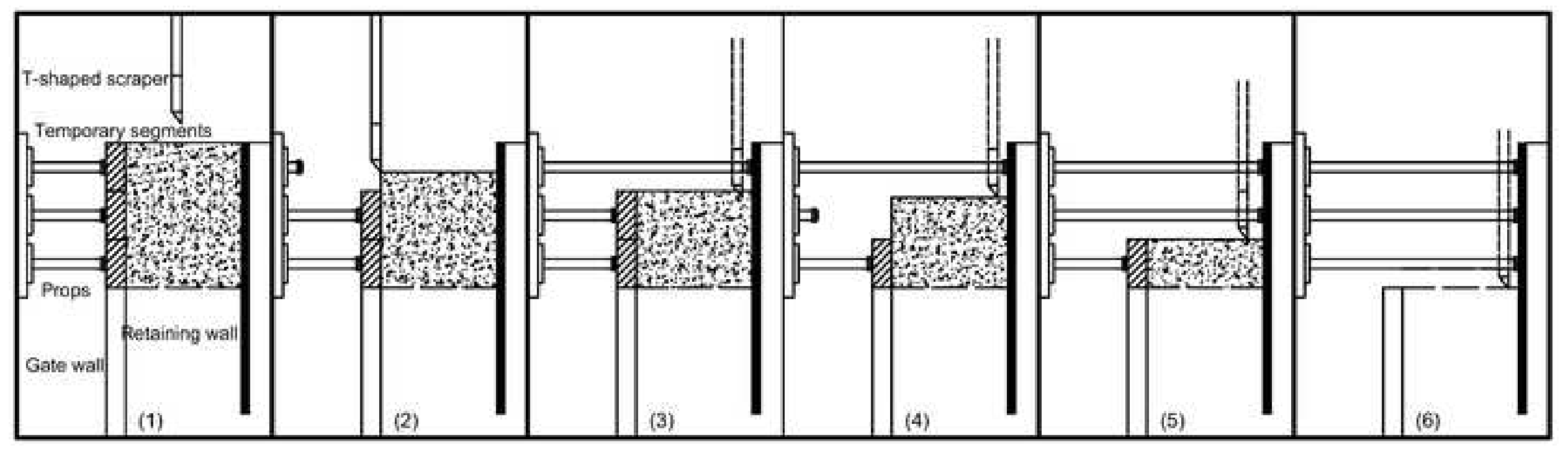




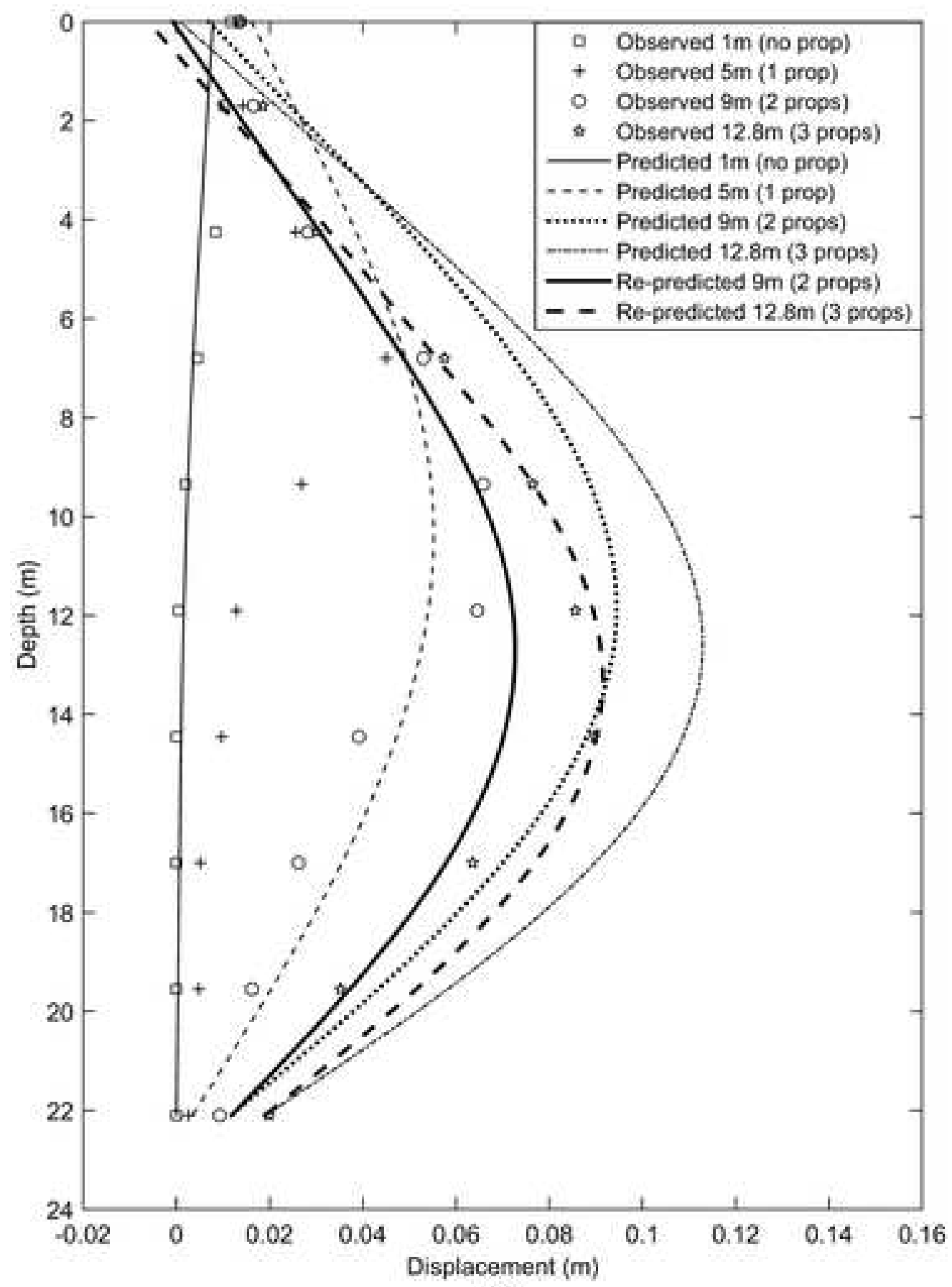

(a)

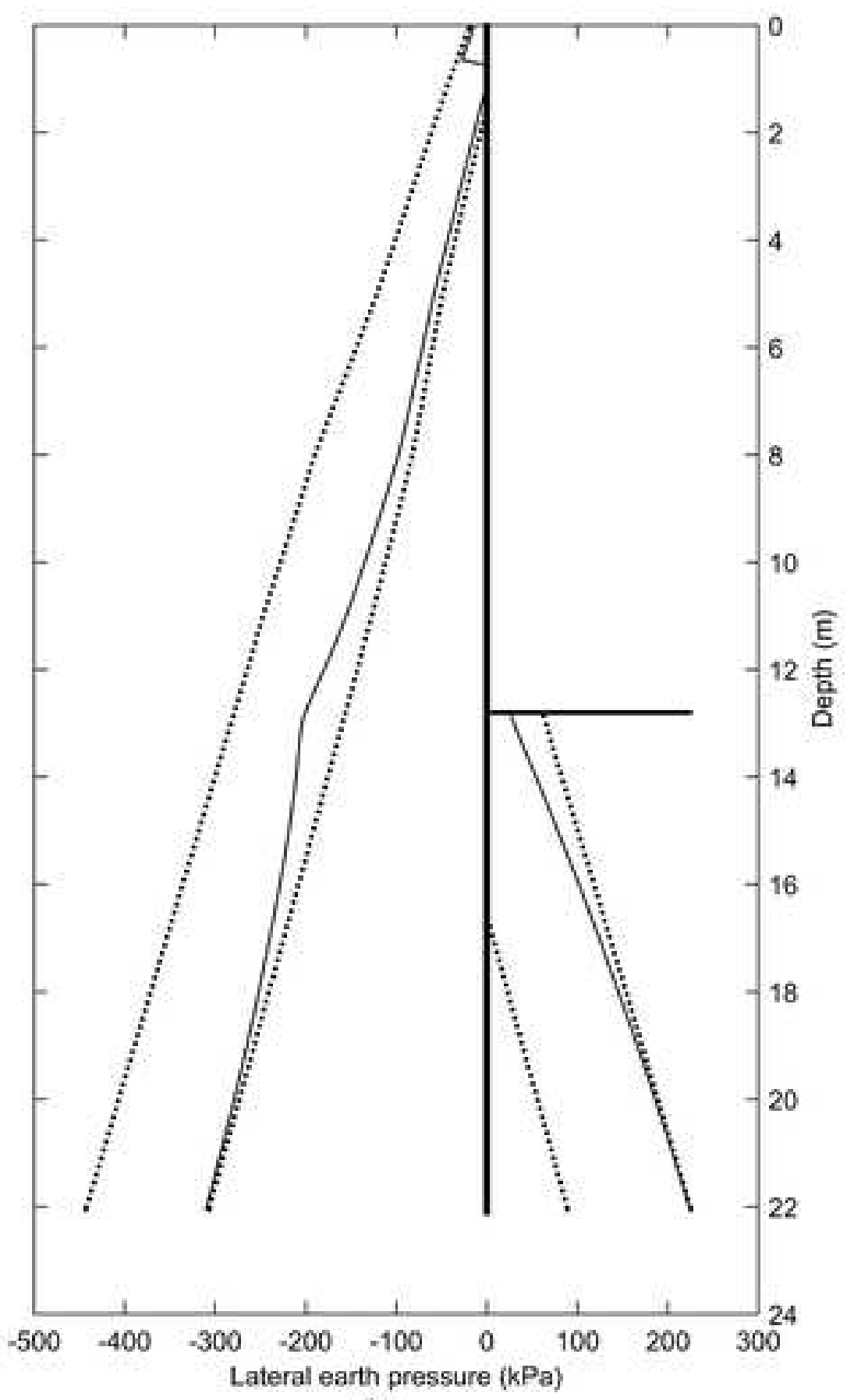

(b) 